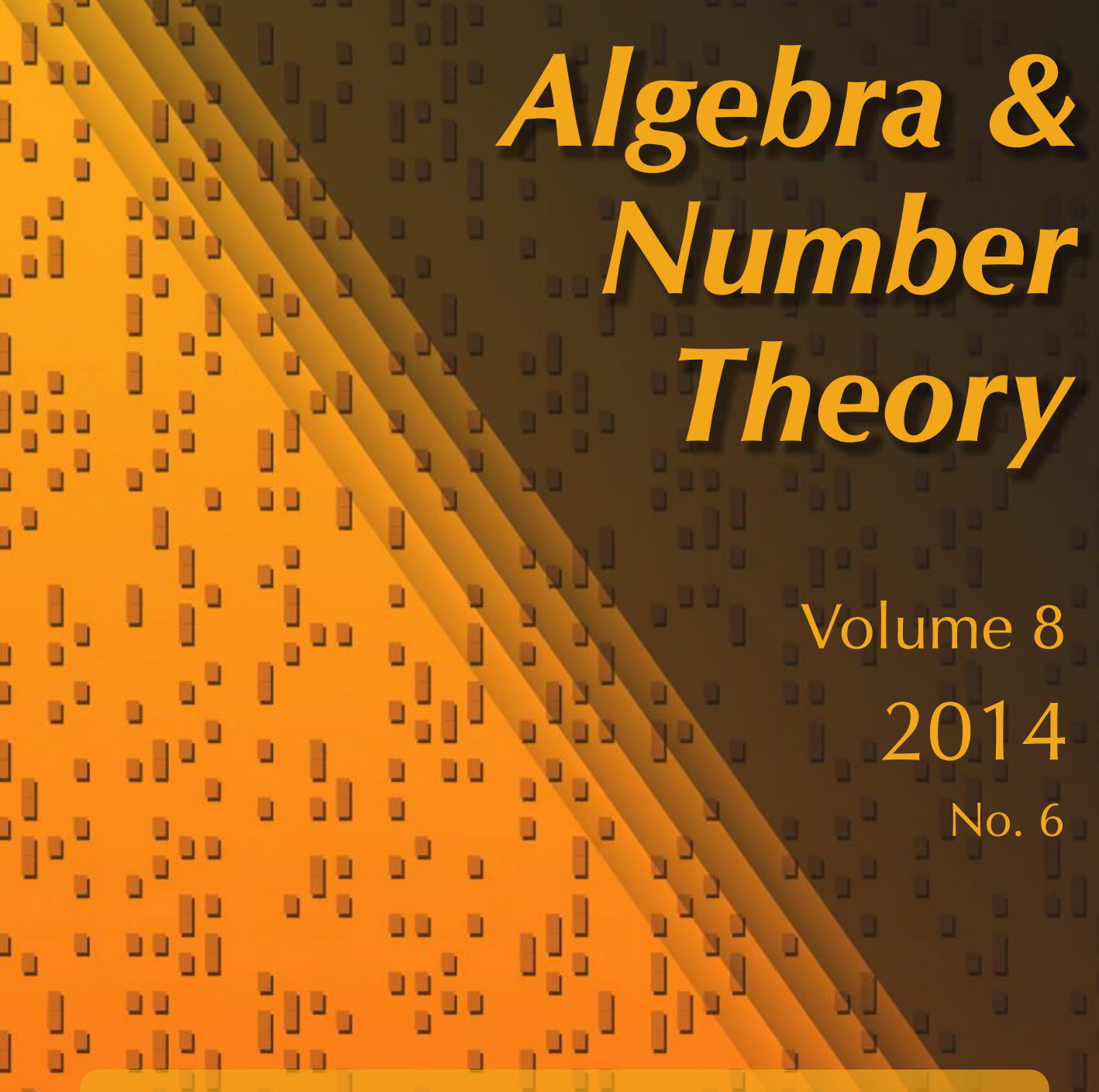




\title{
On moduli spaces for quasitilted algebras
}

\author{
Grzegorz Bobiński
}

\begin{abstract}
We prove that if a quasitilted algebra is tame, then the associated moduli spaces are products of projective spaces. Together with an earlier result of Chindris this gives a geometric characterization of the tame quasitilted algebras. In our proof we use knowledge of the representation theory of the tame quasitilted algebras and a construction of semi-invariants as determinants.
\end{abstract}

Throughout the article $\mathbb{k}$ is an algebraically closed field of characteristic 0 . By $\mathbb{Z}$, $\mathbb{N}$ and $\mathbb{N}_{+}$we denote the sets of integers, nonnegative integers and positive integers, respectively. Finally, if $i, j \in \mathbb{Z}$, then $[i, j]:=\{k \in \mathbb{Z} \mid i \leq k \leq j\}$ (in particular, $[i, j]=\varnothing$ if $i>j)$.

There is a well-known dichotomy for finite-dimensional algebras due to Drozd [1980]: every algebra is either tame or wild, but not both. Here a finite-dimensional algebra is called tame if, for each dimension $d$, the indecomposable $d$-dimensional modules form finitely many one-parameter families. On the other hand, an algebra $\Lambda$ is wild if the classification of $\Lambda$-modules is as difficult as the classification of the pairs of two (noncommuting) endomorphisms of a finite-dimensional vector space (the latter problem is considered to be hopeless).

The above definitions of tame and wild algebras are of a geometric nature. This encourages people to look for characterizations of representation type, which use properties of geometric objects associated with them, for example, module varieties (some results of this type can be found in [Bobiński and Skowroński 1999; Skowroński and Zwara 1998]). In particular, Skowroński and Weyman [2000] have proved that a hereditary algebra $\Lambda$ is tame if and only if all the corresponding rings of semi-invariants are complete intersections. Inspired by this result Chindris [2009; 2011 ; 2013] has initiated a programme, whose aim is to characterize representation type in terms of (rational) invariant theory (see also [Domokos 2011] for an earlier result in this direction). As a result of his studies he and Carroll published the following conjecture, which they attribute to Weyman:

Conjecture [Carroll and Chindris 2012]. Let $\Lambda$ be an algebra. Then the following conditions are equivalent:

MSC2010: primary 16G10; secondary 16G60, 13A50.

Keywords: quasitilted algebra, moduli space, semi-invariant. 
(1) $\Lambda$ is of tame representation type.

(2) For any dimension vector $\boldsymbol{d}$, for any irreducible component $\mathscr{b}$ of the variety $\bmod _{\Lambda}(\boldsymbol{d})$ of $\Lambda$-modules of dimension vector $\boldsymbol{d}$, and for any weight $\theta$ such that $\mathscr{C}_{\theta}^{\mathrm{ss}} \neq \varnothing, M(\mathscr{C})_{\theta}^{\mathrm{ss}}$ is a product of projective spaces.

Here $\mathscr{C}_{\theta}^{\text {ss }}$ denotes the open subset of $\theta$-semistable modules in $\mathscr{C}$ and $\mathcal{M}(\mathscr{C})_{\theta}^{\text {ss }}$ denotes the associated moduli space (see Section 5).

This conjecture has no chance of holding in such generality. Obvious counterexamples are local wild algebras. There is also a counterexample due to Ringel of a triangular (no cycles in the Gabriel quiver) wild algebra, such that all the associated moduli spaces are points.

The aim of this paper is to verify this conjecture for the quasitilted algebras. The quasitilted algebras form an important class of finite-dimensional algebras. Using covering techniques, the study of some classes of algebras can be reduced to the study of quasitilted algebras. In particular, every self-injective algebra of polynomial growth is a socle deformation of an orbit algebra of the repetitive algebra of a tame quasitilted algebra with positive semidefinite Euler form (for more in this direction see the survey article [Skowroński 2006]).

The main result of the paper is the following:

Theorem 1. Let $\Lambda$ be a quasitilted algebra. Then the following conditions are equivalent:

(1) $\Lambda$ is of tame representation type.

(2) For any dimension vector $\boldsymbol{d}$, for any irreducible component $\mathscr{C}$ of $\bmod _{\Lambda}(\boldsymbol{d})$, and for any weight $\theta$ such that $\mathscr{C}_{\theta}^{\mathrm{sS}} \neq \varnothing, \mathcal{M}(\mathscr{C})_{\theta}^{\mathrm{ss}}$ is a product of projective spaces.

The implication $(2) \Longrightarrow(1)$ has been proved for the tilted algebras by Chindris [2013, Proposition 4.1]. In fact, as has been explained to me by Chindris, his proof of the implication (2) $\Longrightarrow(1)$ generalizes to the quasitilted algebras. More precisely, Chindris [2013] used a result of Kerner [1989] stating that every wild tilted algebra has a convex subalgebra which is wild concealed. In the case of the quasitilted algebras one has to use results of Lenzing and Skowroński [1996] (every wild quasitilted algebra has a convex subalgebra which is wild almost concealed-canonical) and Meltzer [1996] (every wild almost concealed-canonical algebra has a convex subcategory which is wild concealed). Thus in the paper we concentrate on the proof of the following theorem:

Theorem 2. Let $\Lambda$ be a tame quasitilted algebra. Then for any dimension vector $\boldsymbol{d}$, for any irreducible component $\mathscr{C}$ of $\bmod _{\Lambda}(\boldsymbol{d})$, and for any weight $\theta$ such that $\mathscr{C}_{\theta}^{\mathrm{ss}} \neq \varnothing, \mathcal{M}(\mathscr{C})_{\theta}^{\mathrm{ss}}$ is a product of projective spaces. 
The paper is organized as follows. In Section 1 we recall basic facts about quivers and their representations. Section 2 is devoted to a short introduction of quasitilted algebras. Next, in Sections 3 and 4 we introduce module varieties and semi-invariants, respectively. Moreover, in Section 4 some reduction results for semi-invariants are obtained. Finally, in Section 5 we recall King's construction of moduli spaces and in Section 6 we prove that the moduli spaces for the tame quasitilted algebras are products of projective spaces.

\section{Quivers and their representations}

In this section we present facts about quivers and their representations, which we use in the paper. As a general background we suggest [Assem et al. 2006; Auslander et al. 1997; Ringel 1984].

By a quiver $Q$ we mean a finite set $Q_{0}$ (called the set of vertices of $Q$ ) together with a finite set $Q_{1}$ (called the set of arrows of $Q$ ) and two maps $s, t: Q_{1} \rightarrow Q_{0}$, which assign to each arrow $\alpha$ its starting vertex $s \alpha$ and its terminating vertex $t \alpha$, respectively. By a path of length $n \in \mathbb{N}_{+}$in a quiver $Q$ we mean a sequence $\sigma=\left(\alpha_{1}, \ldots, \alpha_{n}\right)$ of arrows such that $s \alpha_{i}=t \alpha_{i+1}$ for each $i \in[1, n-1]$. In the above situation we put $\ell \sigma:=n, s \sigma:=s \alpha_{n}$ and $t \sigma:=t \alpha_{1}$. We treat every arrow in $Q$ as a path of length 1 . Moreover, for each vertex $x$ we have a trivial path $\mathbf{1}_{x}$ at $x$ such that $\ell \mathbf{1}_{x}:=0$ and $s \mathbf{1}_{x}:=x=: t \mathbf{1}_{x}$. For the rest of the paper we assume that the considered quivers do not have oriented cycles, where by an oriented cycle we mean a path $\sigma$ of positive length such that $s \sigma=t \sigma$.

Let $Q$ be a quiver. By the path algebra $\mathbb{k} Q$ of $Q$, we mean the vector space with a basis formed by the paths in $Q$ and multiplication induced by the concatenation of paths. If $x$ and $y$ are vertices of $Q$, we put $\mathbb{k} Q(x, y):=\mathbf{1}_{y} \mathbb{k} Q \mathbf{1}_{x}$; i.e., $\mathbb{k} Q(x, y)$ is the space spanned by the paths with the starting vertex $x$ and the terminating vertex $y$. The (finite-dimensional) $\mathbb{k} Q$-modules may be identified with the $\mathbb{k}$-representations of $Q$, where by a $k$-representation of $Q$ we mean $V$ consisting of finite-dimensional k-vector spaces $V(x), x \in Q_{0}$, and $\mathbb{k}$-linear maps $V(\alpha): V(s \alpha) \rightarrow V(t \alpha), \alpha \in Q_{1}$. In particular, if $M$ is a $\mathbb{k} Q$-module and $V$ is the corresponding representation, then $V(x):=\mathbf{1}_{x} M$ for each $x \in Q_{0}$. We will usually identify $\mathbb{k} Q$-modules with the corresponding representations of $\mathbb{k}$. If $V$ and $W$ are representations of a quiver $Q$, then a morphism $\varphi: V \rightarrow W$ is given by linear maps $\varphi(x): V(x) \rightarrow W(x), x \in Q_{0}$, such that $W(\alpha) \varphi(s \alpha)=\varphi(t \alpha) V(\alpha)$ for each $\alpha \in Q_{1}$. We denote the category of $\mathbb{k}$-representations of $Q$ by rep $Q$. If $V$ is a representation, $x, y \in Q_{0}$, and $\omega \in \mathbb{k} Q(x, y)$, then one defines $V(\omega): V(x) \rightarrow V(y)$ in an obvious way. Given a representation $V$ of $Q$ we denote by $\operatorname{dim} V$ its dimension vector, defined by the formula $(\operatorname{dim} V)(x):=\operatorname{dim}_{\mathbb{k}} V(x)$, for $x \in Q_{0}$. Observe that $\operatorname{dim} V \in \mathbb{N} Q_{0}$ for each representation $V$ of $Q$. We call the elements of $\mathbb{N} Q_{0}$ dimension vectors. If $\boldsymbol{d}$ 
is a dimension vector, then we denote by supp $\boldsymbol{d}$ the subquiver of $Q$ induced by the vertices $x$ such that $\boldsymbol{d}(x) \neq 0$. A dimension vector $\boldsymbol{d}$ is called connected if the quiver supp $\boldsymbol{d}$ is connected. A dimension vector $\boldsymbol{d}$ is called sincere if supp $\boldsymbol{d}=Q$.

By a bound quiver $(Q, I)$ we mean a quiver $Q$ together with an ideal $I$ of $\mathbb{k} Q$ such that $I \subseteq\left\langle Q_{1}\right\rangle^{2}$, where by $\left\langle Q_{1}\right\rangle$ we denote the ideal of $\mathbb{k} Q$ generated by the arrows. Given a bound quiver $(Q, I)$, we call the algebra $\mathbb{k} Q / I$ the path algebra of $(Q, I)$. Note that if $(Q, I)$ is a bound quiver, then the $\mathbb{k} Q / I$-modules may be identified with the representations $V$ of $Q$ such that $V(\omega)=0$ for each $\omega \in I \cap\left(\bigcup_{x, y \in Q_{0}} \mathbb{k} Q(x, y)\right)$. If $\Lambda$ is the path algebra of a bound quiver $(Q, I)$, then we call $Q$ the Gabriel quiver of $\Lambda$. Gabriel proved that (up to isomorphism) $Q$ is uniquely determined by $\Lambda$. Moreover, Gabriel's theorem implies that each quasitilted algebra is Morita equivalent to the path algebra of a bound quiver (since we only consider quivers without oriented cycles, we also need [Happel et al. 1996, Proposition III.1.1(b)] for this result). Thus from now on all algebras considered are the path algebras of bound quivers. Observe that if $J$ is an ideal in an algebra $\Lambda$, then the Gabriel quiver of $\Lambda / J$ is a subquiver of the Gabriel quiver of $\Lambda$ (here this is important that there are no oriented cycles in the considered quivers). If $(Q, I)$ is a bound quiver, then an algebra $\Lambda^{\prime}$ is called a convex subalgebra of $\mathbb{k} Q / I$ if there exists a convex subquiver $Q^{\prime}$ of $Q$ such that $\Lambda^{\prime}=\mathbb{k}_{k} Q^{\prime} /\left(I \cap \mathbb{k} Q^{\prime}\right)$. Recall that a subquiver $Q^{\prime}$ of $Q$ is called convex if for every path $\left(\alpha_{1}, \ldots, \alpha_{n}\right)$ in $Q$ with $s \alpha_{n}, t \alpha_{1} \in Q_{0}^{\prime}$ we have $\alpha_{i} \in Q_{1}^{\prime}$ for each $i \in[1, n]$ (and, consequently, $s \alpha_{i} \in Q_{0}^{\prime}$ for each $\left.i \in[1, n-1]\right)$.

Let $\Lambda$ be an algebra with Gabriel quiver $Q$. For a vertex $x$ of $Q$ we put $P_{\Lambda}(x):=\Lambda \mathbf{1}_{x}$. Then $P_{\Lambda}(x)$ is an indecomposable projective $\Lambda$-module and every indecomposable projective $\Lambda$-module is (up to isomorphism) of this form. If $V$ is a $\Lambda$-module, then $\operatorname{Hom}_{\Lambda}\left(P_{\Lambda}(x), V\right)=V(x)$ for each $x \in Q_{0}$. In particular, $\operatorname{Hom}_{\Lambda}\left(P_{\Lambda}(x), P_{\Lambda}(y)\right)=\mathbb{k} Q(y, x)$ for any $x, y \in Q_{0}$. Moreover, if $x, y \in Q_{0}$, $\omega \in \mathbb{k}(y, x)$ and $V$ is a $\Lambda$-module, then

$$
\operatorname{Hom}_{\Lambda}(\omega, V): \operatorname{Hom}_{\Lambda}\left(P_{\Lambda}(y), V\right) \rightarrow \operatorname{Hom}_{\Lambda}\left(P_{\Lambda}(x), V\right)
$$

is just $V(\omega): V(y) \rightarrow V(x)$.

For an algebra $\Lambda$ we denote by $\bmod \Lambda$ the category of $\Lambda$-modules. Next, if $\Lambda$ is an algebra and $\Lambda^{\mathrm{op}}$ is the opposite algebra of $\Lambda$, then we denote by $\mathrm{D}_{\Lambda}$ the duality $\bmod \Lambda \rightarrow \bmod \Lambda^{\mathrm{op}}$ given by

$$
\mathrm{D}_{\Lambda}(M):=\operatorname{Hom}_{k}(M, k) \quad(M \in \bmod \Lambda) .
$$

Finally, for an algebra $\Lambda$ we denote by $\tau_{\Lambda}$ the corresponding Auslander-Reiten translation, which assigns to each $\Lambda$-module $M$ another $\Lambda$-module $\tau_{\Lambda} M$ (see [Assem et al. 2006, Section IV.2] for a definition). We will need the following consequence of the Auslander-Reiten formula [Assem et al. 2006, Theorem IV.2.13]: if $M$ and $N$ 
are $\Lambda$-modules and $\operatorname{pdim}_{\Lambda} M \leq 1$, then

$$
\operatorname{dim}_{\mathbb{k}} \operatorname{Ext}_{\Lambda}^{1}(M, N)=\operatorname{dim}_{\mathbb{k}} \operatorname{Hom}_{\Lambda}\left(N, \tau_{\Lambda} M\right) .
$$

Let $\Lambda$ be an algebra with Gabriel quiver $Q$. Since there are no cycles in $Q$, gldim $\Lambda<\infty$. Consequently, we may define the bilinear form $\langle-,-\rangle_{\Lambda}$ : $\mathbb{Z}^{Q_{0}} \times \mathbb{Z}^{Q_{0}} \rightarrow \mathbb{Z}$ by the condition

$$
\langle\operatorname{dim} M, \operatorname{dim} N\rangle_{\Lambda}=\sum_{i \in \mathbb{N}} \operatorname{dim}_{k} \operatorname{Ext}_{\Lambda}^{i}(M, N)
$$

for all $\Lambda$-modules $M$ and $N$. We denote the corresponding quadratic form, called the Euler form, by $\chi_{\Lambda}$.

\section{Quasitilted algebras}

A module $T$ over an algebra $\Lambda$ is called tilting if $\operatorname{pdim}_{\Lambda} T \leq 1, \operatorname{Ext}_{\Lambda}^{1}(T, T)=0$, and $T$ is a direct sum of $n$ pairwise nonisomorphic indecomposable $\Lambda$-modules, where $n$ is the number of vertices of the Gabriel quiver of $\Lambda$. By a tilted algebra we mean the opposite algebra of the endomorphism algebra of a tilting module over the path algebra of a quiver.

An algebra $\Lambda$ is called quasitilted if $\Lambda$ is the opposite algebra of the endomorphism algebra of a tilting object in a connected hereditary abelian $\mathbb{k}$-category with finite-dimensional homomorphism and extension spaces. Equivalently, gldim $\Lambda \leq 2$ and either $\operatorname{pdim}_{\Lambda} X \leq 1$ or $\operatorname{idim}_{\Lambda} X \leq 1$ for each indecomposable $\Lambda$-module $X$ (see [Happel et al. 1996, Theorem 2.3]). Two prominent examples of quasitilted algebras are the tilted algebras introduced above and the Ringel canonical algebras $\Lambda(\boldsymbol{m}, \lambda)$, where $\boldsymbol{m}=\left(m_{1}, \ldots, m_{n}\right), n \geq 3$, is a sequence of integers greater than 1 and $\lambda=\left(\lambda_{3}, \ldots, \lambda_{n}\right)$. In the above situation $\Lambda(\boldsymbol{m}, \lambda)$ is the path algebra of the quiver

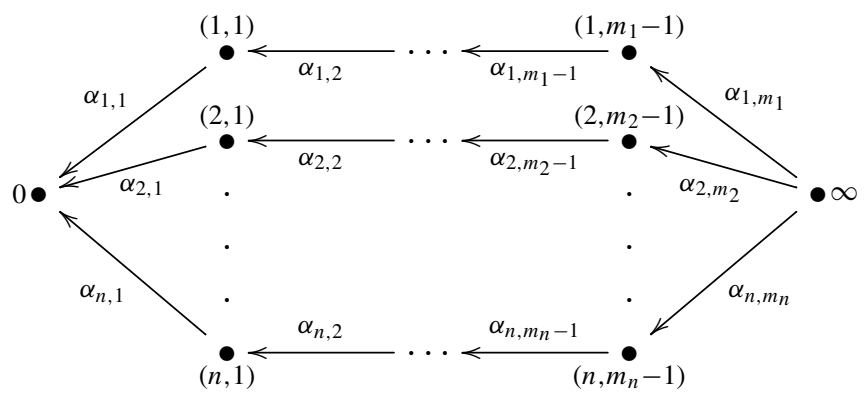

modulo the ideal generated by the relations

$$
\alpha_{1,1} \cdots \alpha_{1, m_{1}}+\lambda_{i} \alpha_{2,1} \cdots \alpha_{2, m_{2}}-\alpha_{i, 1} \cdots \alpha_{i, m_{i}}, \quad i \in[3, n] .
$$

Due to [Happel 2001, Theorem 3.1] every quasitilted algebra is either a tilted algebra or is of canonical type (i.e., is derived equivalent to a canonical algebra). 
The structure of the module categories over tilted algebras has been investigated in [Kerner 1989], while the structure of the module categories over quasitilted algebras of canonical type has been studied in [Lenzing and Skowroński 1996]. We also refer to [Skowroński 1998] for a characterization of the tame quasitilted algebras and to [Ringel 1984] for a description of the module categories over socalled tubular algebras, which form an important subclass of the tame quasitilted algebras. We list some consequences of these investigations.

Let $\Lambda$ be a tame quasitilted algebra with Gabriel quiver $Q$. If $\boldsymbol{d}$ is a dimension vector, then there exists an indecomposable $\Lambda$-module with dimension vector $\boldsymbol{d}$ if and only if $\boldsymbol{d}$ is a root of $\chi_{\Lambda}$, i.e., $\boldsymbol{d}$ is a connected nonzero dimension vector such that $\chi_{\Lambda}(\boldsymbol{d}) \in\{0,1\}$. We call a root $\boldsymbol{d}$ isotropic if $\chi_{\Lambda}(\boldsymbol{d})=0$. We call a root $\boldsymbol{d}$ a Schur root if there exists a $\Lambda$-module $X$ (necessarily indecomposable) with dimension vector $\boldsymbol{d}$ and trivial endomorphism algebra. Let $\boldsymbol{d}_{1}$ and $\boldsymbol{d}_{2}$ be two isotropic roots with supp $\boldsymbol{d}_{1} \cap \operatorname{supp} \boldsymbol{d}_{2} \neq \varnothing$. Then $\operatorname{Hom}_{\Lambda}\left(X_{1}, X_{2}\right) \neq 0$ for all indecomposable $\Lambda$-modules $X_{1}$ and $X_{2}$ with $\operatorname{dim} X_{1}=d_{1}$ and $\operatorname{dim} X_{2}=$ $\boldsymbol{d}_{2}$, or $\operatorname{Hom}_{\Lambda}\left(X_{2}, X_{1}\right) \neq \varnothing$ for all indecomposable $\Lambda$-modules $X_{1}$ and $X_{2}$ with $\operatorname{dim} X_{1}=d_{1}$ and $\operatorname{dim} X_{2}=d_{2}$, or $d_{1}$ and $d_{2}$ are multiplicities of the same isotropic Schur root.

Now assume that $\Lambda$ is a canonical algebra. The indecomposable $\Lambda$-modules can be divided into three classes, $\mathscr{L}_{\Lambda}, \mathscr{T}_{\Lambda}$ and $\mathscr{R}_{\Lambda}$ : the class $\mathscr{L}_{\Lambda}$ is formed by the indecomposable $\Lambda$-modules $X$ such that $\operatorname{dim} X(0)>\operatorname{dim} X(\infty)$, the class $\mathscr{T}_{\Lambda}$ is formed by the indecomposable $\Lambda$-modules $X$ such that $\operatorname{dim} X(0)=\operatorname{dim} X(\infty)$, and the class $\mathscr{R}_{\Lambda}$ is formed by the indecomposable $\Lambda$-modules $X$ such that $\operatorname{dim} X(0)<$ $\operatorname{dim} X(\infty)$. An algebra $\Lambda$ is called (almost) concealed-canonical if $\Lambda$ is the opposite algebra of the endomorphism algebra of a tilting module $T$, which is a direct sum of indecomposable modules from $\mathscr{L}_{\Lambda}\left(\mathscr{L}_{\Lambda} \cup \mathscr{T}_{\Lambda}\right.$, respectively).

\section{Module varieties}

Let $\Lambda$ be the path algebra of a bound quiver $(Q, I)$. For a dimension vector $\boldsymbol{d}$ we denote by $\bmod _{\Lambda}(\boldsymbol{d})$ the set of representations $M$ of $(Q, I)$ (recall that we identify the $\Lambda$-modules with the representations of $(Q, I))$ such that $M(x)=\mathbb{k}^{d(x)}$ for each $x \in Q_{0}$. This set can be naturally identified with a closed subset of the affine space $\operatorname{rep}_{Q}(\boldsymbol{d}):=\prod_{\alpha \in Q_{1}} \mathbb{M}(\boldsymbol{d}(t \alpha), \boldsymbol{d}(s \alpha))$; thus it has the structure of an affine variety (note that under this identification $\operatorname{rep}_{Q}(\boldsymbol{d})=\bmod _{\mathbb{k} Q}(\boldsymbol{d})$ ). The reductive group $\mathrm{GL}(\boldsymbol{d}):=\prod_{x \in Q_{0}} \mathrm{GL}(\boldsymbol{d}(x))$ acts on $\bmod _{\Lambda}(\boldsymbol{d})$ via

$$
(g * M)(\alpha):=g(t \alpha) \cdot M(\alpha) \cdot g(s \alpha)^{-1} \quad\left(g \in \mathrm{GL}(\boldsymbol{d}), \alpha \in Q_{1}\right) .
$$

If $M \in \bmod _{\Lambda}(d)$, then we denote its orbit with respect to this action by $\mathcal{O}(M)$. One

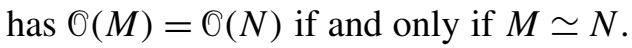


Let $\mathscr{C}_{1}$ and $\mathscr{C}_{2}$ be closed irreducible subsets of varieties $\bmod _{\Lambda}\left(\boldsymbol{d}_{1}\right)$ and $\bmod { }_{\Lambda}\left(\boldsymbol{d}_{2}\right)$, respectively. By $\mathscr{C}_{1} \oplus \mathscr{C}_{2}$ we denote the closure of the set consisting of all $M \in$ $\bmod _{\Lambda}\left(\boldsymbol{d}_{1}+\boldsymbol{d}_{2}\right)$ such that $M \simeq M_{1} \oplus M_{2}$ for some $M_{1} \in \mathscr{C}_{1}$ and $M_{2} \in \mathscr{C}_{2}$. In the above situation we call $\mathscr{C}_{1}$ and $\mathscr{C}_{2}$ summands of $\mathscr{C}$.

An irreducible component $\mathscr{C}$ of $\bmod _{\Lambda}(\boldsymbol{d})$ is called indecomposable if the indecomposable modules in $\mathscr{C}$ form a dense subset of $\mathscr{C}$. If $\mathscr{C}$ is an irreducible component of $\bmod _{\Lambda}(\boldsymbol{d})$, then there exist uniquely determined (up to ordering) indecomposable irreducible components $\mathscr{b}_{1}, \ldots, \mathscr{C}_{n}$ of $\mathscr{C}$ such that

$$
\mathscr{C}=\mathscr{b}_{1} \oplus \cdots \oplus \mathscr{b}_{n}
$$

[Crawley-Boevey and Schröer 2002, Theorem 1.1] (see also [de la Peña 1991]). We call the above presentation the generic decomposition of $\mathscr{C}$. Moreover, if, for $i \in[1, n], \mathscr{C}_{i} \subseteq \bmod _{\Lambda}\left(\boldsymbol{d}_{i}\right)$, then we call $\boldsymbol{d}_{1}, \ldots, \boldsymbol{d}_{n}$ the generic summands of $\boldsymbol{d}$ at $\mathscr{C}$.

Now we present a description of the indecomposable irreducible components in the case of the tame quasitilted algebras, which follows from [Bobiński and Skowroński 1999]. First, if $\boldsymbol{d}$ is a dimension vector, then there is at most one indecomposable irreducible component of $\bmod _{\Lambda}(\boldsymbol{d})$. Thus if it exists we denote it by $\mathscr{C}(\boldsymbol{d})$. Moreover, there exists an indecomposable irreducible component of $\bmod _{\Lambda}(\boldsymbol{d})$ if and only if $\boldsymbol{d}$ is a Schur root. Moreover, if $\boldsymbol{d}$ is not isotropic, then $\mathscr{C}(\boldsymbol{d})$ is an orbit closure, i.e., there exists a $\Lambda$-module $M$ such that $\mathscr{C}(\boldsymbol{d})=\overline{\mathrm{O}(M)}$.

\section{Semi-invariants}

Let $Q$ be a quiver, $\boldsymbol{d}$ a dimension vector, and $\mathscr{C}$ a GL(d)-invariant closed subset of $\operatorname{rep}_{Q}(\boldsymbol{d})$. The action of $\mathrm{GL}(\boldsymbol{d})$ on $\mathscr{C}$ induces an action on the coordinate ring $\mathbb{k}[\mathscr{C}]$ via

$$
(g * f)(M):=f\left(g^{-1} * M\right) \quad(g \in \mathrm{GL}(\boldsymbol{d}), f \in \mathbb{k}[\mathscr{C}], M \in \mathscr{C}) .
$$

If $\mathscr{C}$ is irreducible, then there is a unique closed orbit in $\mathscr{b}$, that of the semisimple module with dimension vector $\boldsymbol{d}$, hence there are only trivial GL $(\boldsymbol{d})$-invariant regular functions on $\mathscr{C}$, i.e., $\mathbb{k}_{[}[\mathfrak{b}]^{\mathrm{GL}(d)}=\mathbb{k}$. However, one may still have nontrivial semiinvariants. A regular function $f \in \mathbb{k}[\mathscr{C}]$ is called a semi-invariant of weight $\theta \in \mathbb{Z}^{Q_{0}}$ if

$$
g * f=\chi^{\theta}(g) \cdot f
$$

for each $g \in \mathrm{GL}(\boldsymbol{d})$. Here $\chi^{\theta}: \operatorname{GL}(\boldsymbol{d}) \rightarrow \mathbb{k}^{\times}$is given by

$$
\chi^{\theta}(g):=\prod_{x \in Q_{0}} \operatorname{det}^{\theta\left(\boldsymbol{e}_{x}\right)}(g(x)) \quad(g \in \mathrm{GL}(\boldsymbol{d})),
$$


where $\boldsymbol{e}_{x}, x \in Q_{0}$, are the standard basis vectors of $\mathbb{Z}^{Q_{0}}$. We denote the space of semi-invariants of weight $\theta$ by SI $[\mathscr{C}]_{\theta}$. One easily observes that $\theta(\boldsymbol{d})=0$ provided $\mathrm{SI}[\mathscr{C}]_{\theta} \neq 0$.

We present a method of constructing semi-invariants, which in the case of quivers is due to Schofield [1991], and has been generalized to the case of bound quivers independently by Derksen and Weyman [2002] and Domokos [2002] (we also refer to the latter two articles for proofs). Let $Q$ be a quiver and $\boldsymbol{d}$ a dimension vector. Fix sequences $\boldsymbol{x}=\left(x_{1}, \ldots, x_{n}\right)$ and $\boldsymbol{y}=\left(y_{1}, \ldots, y_{m}\right)$ of vertices of $Q$. Put

$$
\mathbb{k} Q(\boldsymbol{x}, \boldsymbol{y}):=\prod_{\substack{i \in[1, n] \\ j \in[1, m]}} \mathbb{k} Q\left(x_{i}, y_{j}\right) .
$$

If $\phi=\left(\phi_{i, j}\right)_{i \in[1, n], j \in[1, m]} \in \mathbb{k} Q(\boldsymbol{x}, \boldsymbol{y})$ and $M \in \operatorname{rep}_{Q}(\boldsymbol{d})$ for a dimension vector $\boldsymbol{d}$, then we obtain a map

$$
M(\phi):=\left[M\left(\phi_{i, j}\right)\right]_{\substack{i \in[1, n] \\ j \in[1, m]}}: M(\boldsymbol{x}):=\bigoplus_{i \in[1, n]} M\left(x_{i}\right) \rightarrow M(\boldsymbol{y}):=\bigoplus_{j \in[1, m]} M\left(y_{j}\right) .
$$

If, in addition, $\sum_{i \in[1, n]} \boldsymbol{d}\left(x_{i}\right)=\sum_{j \in[1, m]} \boldsymbol{d}\left(y_{j}\right)$, then we may define a regular function $c_{\boldsymbol{d}}^{\phi}: \operatorname{rep}_{Q}(\boldsymbol{d}) \rightarrow \mathbb{k}$ by

$$
c_{d}^{\phi}(M):=\operatorname{det} M(\phi) \quad\left(M \in \operatorname{rep}_{Q}(\boldsymbol{d})\right) .
$$

Then $c_{\boldsymbol{d}}^{\phi}$ is a semi-invariant of weight $\theta^{\phi}$, where

$$
\theta^{\phi}(\boldsymbol{c}):=\sum_{i \in[1, n]} \boldsymbol{c}\left(x_{i}\right)-\sum_{j \in[1, m]} \boldsymbol{c}\left(y_{j}\right) \quad\left(\boldsymbol{c} \in \mathbb{Z}^{Q_{0}}\right)
$$

(note that, in particular, $\theta^{\phi}(\boldsymbol{d})=0$ ). If $\mathscr{C}$ is a $\operatorname{GL}(\boldsymbol{d})$-invariant closed subset of $\operatorname{rep}_{Q}(\boldsymbol{d})$, then we denote the restriction $\left.c_{\boldsymbol{d}}^{\phi}\right|_{\mathscr{C}}$ of $c_{\boldsymbol{d}}^{\phi}$ to $\mathscr{C}$ by $c_{\mathscr{C}}^{\phi}$.

We list some obvious consequences (cf. [Derksen and Weyman 2000, Lemma 1]).

Lemma 4.1. Let $\boldsymbol{x}$ and $\boldsymbol{y}$ be sequences of vertices of a quiver $Q, \phi \in \mathbb{k} Q(\boldsymbol{x}, \boldsymbol{y})$, $\boldsymbol{d}$ a dimension vector such that $\theta^{\phi}(\boldsymbol{d})=0$, and $M=M_{1} \oplus M_{2} \in \bmod _{\Lambda}(\boldsymbol{d})$.

(1) If $\theta^{\phi}\left(\operatorname{dim} M_{1}\right) \neq 0$ (hence, equivalently, $\left.\theta^{\phi}\left(\operatorname{dim} M_{2}\right) \neq 0\right)$, then $c_{\boldsymbol{d}}^{\phi}(M)=0$.

(2) If $\theta^{\phi}\left(\operatorname{dim} M_{1}\right)=0$ (hence, equivalently, $\left.\theta^{\phi}\left(\operatorname{dim} M_{2}\right)=0\right)$, then $c_{d}^{\phi}(M)=$ $c_{\operatorname{dim} M_{1}}^{\phi}\left(M_{1}\right) \cdot c_{\operatorname{dim} M_{2}}^{\phi}\left(M_{2}\right)$.

If we have sequences $\boldsymbol{x}, \boldsymbol{x}^{\prime}, \boldsymbol{y}$ and $\boldsymbol{y}^{\prime}$ of vertices of a quiver $Q, \phi \in \mathbb{k}(\boldsymbol{x}, \boldsymbol{y})$ and $\phi^{\prime} \in \mathbb{k}\left(\boldsymbol{x}^{\prime}, \boldsymbol{y}^{\prime}\right)$, then we may define an element $\phi \oplus \phi^{\prime} \in \mathbb{k} Q\left(\boldsymbol{x} \cdot \boldsymbol{x}^{\prime}, \boldsymbol{y} \cdot \boldsymbol{y}^{\prime}\right)$ in the obvious way, where $\boldsymbol{x} \cdot \boldsymbol{x}^{\prime}$ and $\boldsymbol{y} \cdot \boldsymbol{y}^{\prime}$ are the concatenations of the respective sequences. Observe that

$$
M\left(\phi \oplus \phi^{\prime}\right)=\left[\begin{array}{cc}
M(\phi) & 0 \\
0 & M\left(\phi^{\prime}\right)
\end{array}\right]: M(\boldsymbol{x}) \oplus M\left(\boldsymbol{x}^{\prime}\right) \rightarrow M(\boldsymbol{y}) \oplus M\left(\boldsymbol{y}^{\prime}\right)
$$


for each $M \in \operatorname{rep}_{Q}(\boldsymbol{d})$. Consequently, we get the following.

Lemma 4.2. Let $\boldsymbol{x}, \boldsymbol{x}^{\prime}, \boldsymbol{y}$ and $\boldsymbol{y}^{\prime}$ be sequences of vertices of a quiver $Q, \phi \in \mathbb{k}(\boldsymbol{x}, \boldsymbol{y})$ and $\phi^{\prime} \in \mathbb{k}\left(\boldsymbol{x}^{\prime}, \boldsymbol{y}^{\prime}\right)$. If $\boldsymbol{d}$ is a dimension vector and $\theta^{\phi}(\boldsymbol{d})=0=\theta^{\phi^{\prime}}(\boldsymbol{d})$, then

$$
c_{\boldsymbol{d}}^{\phi \oplus \phi^{\prime}}=c_{\boldsymbol{d}}^{\phi} \cdot c_{\boldsymbol{d}}^{\phi^{\prime}}
$$

In particular, $c_{\boldsymbol{d}}^{\phi \oplus \phi^{\prime}}$ is a semi-invariant of weight $\theta^{\phi}+\theta^{\phi^{\prime}}$.

We can interpret the above construction using projective presentations. Let $\Lambda$ be a factor algebra of $\mathbb{k} Q$ for a quiver $Q$. As above, let $\boldsymbol{x}=\left(x_{1}, \ldots, x_{n}\right)$ and $\boldsymbol{y}=\left(y_{1}, \ldots, y_{m}\right)$ be sequences of vertices of a quiver $Q$, and $\phi \in \mathbb{k} Q(\boldsymbol{x}, \boldsymbol{y})$. If we put

$$
P_{\Lambda}(\boldsymbol{x}):=\bigoplus_{i \in[1, n]} P_{\Lambda}\left(x_{i}\right) \quad \text { and } \quad P_{\Lambda}(\boldsymbol{y}):=\bigoplus_{j \in[1, m]} P_{\Lambda}\left(y_{j}\right)
$$

then we may view $\phi$ as a map $P_{\Lambda}(\boldsymbol{y}) \rightarrow P_{\Lambda}(\boldsymbol{x})$ (note that every map between projective $\Lambda$-modules is of this form, for some $\boldsymbol{x}, \boldsymbol{y}$ and $\phi$ ). Observe that

$$
\theta^{\phi}(\operatorname{dim} M)=\operatorname{dim}_{\mathbb{k}} \operatorname{Hom}_{\Lambda}\left(P_{\Lambda}(\boldsymbol{x}), M\right)-\operatorname{dim}_{\mathbb{k}} \operatorname{Hom}_{\Lambda}\left(P_{\Lambda}(\boldsymbol{y}), M\right)
$$

for each $\Lambda$-module $M$. If $M \in \bmod _{\Lambda}(\boldsymbol{d})$ for a dimension vector $\boldsymbol{d}$, then $M(\phi)$ may be identified with the induced map

$$
\operatorname{Hom}_{\Lambda}(\phi, M): \operatorname{Hom}_{\Lambda}\left(P_{\Lambda}(\boldsymbol{x}), M\right) \rightarrow \operatorname{Hom}_{\Lambda}\left(P_{\Lambda}(\boldsymbol{y}), M\right) .
$$

This implies in particular that if $\theta^{\phi}(\boldsymbol{d})=0$, then $c_{\boldsymbol{d}}^{\phi}(M) \neq 0$ if and only if $\operatorname{Hom}_{\Lambda}(\operatorname{Coker} \phi, M)=0$. We associate a semi-invariant with Coker $\phi$ (independently of $\phi$ ). In order to simplify the presentation, we make some additional assumptions.

Let $\Lambda$ be an algebra with Gabriel quiver $Q$. Moreover, let $V$ be a $\Lambda$-module with projective dimension at most 1. If $\phi: Q \rightarrow P$ is a projective presentation of $V$ such that $\phi$ is a monomorphism, then $\theta^{\phi}=\langle\operatorname{dim} V,-\rangle_{\Lambda}$, hence is independent of $\phi$. We denote this weight by $\theta^{V}$. Moreover, if $\theta^{V}(\boldsymbol{d})=0$, and $\phi$ and $\phi^{\prime}$ are projective presentations of $V$ such that $\phi$ and $\phi^{\prime}$ are monomorphisms, then $c_{\bmod _{\Lambda}(\boldsymbol{d})}^{\phi}$ and $c_{\bmod _{\Lambda}(\boldsymbol{d})}^{\phi^{\prime}}$ coincide up to a nonzero scalar. Thus we may define $c_{\Lambda, \boldsymbol{d}}^{V} \in \mathbb{k}\left[\bmod _{\Lambda}(\boldsymbol{d})\right]$ by $c_{\Lambda, \boldsymbol{d}}^{V}:=c_{\bmod _{\Lambda}(\boldsymbol{d})}^{\phi}$, where $\phi$ is a chosen projective presentation of $V$. Then $c_{\Lambda, \boldsymbol{d}}^{V}$ is a semi-invariant of weight $\theta^{V}$ and $c_{\Lambda, d}^{V}(M) \neq 0$ if and only if $\operatorname{Hom}_{\Lambda}(V, M)=0$ or, equivalently, $\operatorname{Hom}_{\Lambda}\left(M, \tau_{\Lambda} V\right)=0$ (for the latter statement we need (1)). If $\mathscr{C}$ is a closed GL $(\boldsymbol{d})$-invariant subset of $\bmod _{\Lambda}(\boldsymbol{d})$, then we denote by $c_{\Lambda, \mathscr{C}}^{V}$ the restriction of $c_{\Lambda, d}^{V}$ to $\mathscr{C}$.

It will be often useful to associate semi-invariants to modules of projective dimension at most 1 in a "regular" way. Thus assume in addition that $c$ is a dimension vector such that $\mathscr{P}_{\Lambda}(\boldsymbol{c}) \neq \varnothing$, where $\mathscr{P}_{\Lambda}(\boldsymbol{c})$ is the subset of $\bmod _{\Lambda}(\boldsymbol{c})$ consisting of the modules of projective dimension at most 1 . Let $\boldsymbol{x}$ be a sequence of vertices 
of $Q$ such that $P_{\Lambda}(x)=\bigoplus_{x \in Q_{0}} P_{\Lambda}(x)^{c(x)}$. Since there exists an epimorphism $P_{\Lambda}(\boldsymbol{x}) \rightarrow V$ for each $\Lambda$-module $V$ with dimension vector $\boldsymbol{c}$ and $\mathscr{P}_{\Lambda}(\boldsymbol{c}) \neq \varnothing$, there exists a sequence $\boldsymbol{y}$ of vertices of $Q$ such that $\operatorname{dim} P_{\Lambda}(\boldsymbol{x})-\operatorname{dim} P_{\Lambda}(\boldsymbol{y})=\boldsymbol{c}$. Let $\mathscr{X}_{\Lambda}(\boldsymbol{c}) \subseteq \mathbb{k} Q(\boldsymbol{x}, \boldsymbol{y})$ be the set of monomorphisms $P_{\Lambda}(\boldsymbol{y}) \hookrightarrow P_{\Lambda}(\boldsymbol{x})$. Obviously, if $\phi \in \mathscr{X}_{\Lambda}(\boldsymbol{c})$, then Coker $\phi \in \mathscr{P}_{\Lambda}(\boldsymbol{c})$. On the other hand, if $V \in \mathscr{P}_{\Lambda}(\boldsymbol{c})$, then there exists $\phi \in \mathscr{X}_{\Lambda}(\boldsymbol{d})$ such that Coker $\phi \simeq V$. In fact we have even more:

Lemma 4.3. Let $\Lambda$ be an algebra and $\boldsymbol{c}$ a dimension vector. If $\mathscr{V}$ is a nonempty open subset of $\mathscr{P}_{\Lambda}(\boldsymbol{c})$ and

$$
\mathscr{U}:=\left\{\phi \in \mathscr{X}_{\Lambda}(\boldsymbol{c}) \mid \operatorname{Coker} \phi \in \mathscr{V}\right\},
$$

then $\mathcal{U}$ is a nonempty open subset of $\mathscr{X}_{\Lambda}(\boldsymbol{c})$.

Proof. Let $P:=\bigoplus_{x \in Q_{0}} P_{\Lambda}(x)^{c(x)}$. Let $\mathscr{Y}_{\Lambda}(\boldsymbol{c})$ be the set of $\psi=(\psi(x))_{x \in Q_{0}}$ such that, for each $x \in Q_{0}, \psi(x): P(x) \rightarrow \mathbb{k}^{c(x)}$ is a linear map. We denote by $\mathscr{L}_{\Lambda}(\boldsymbol{c})$ the set of pairs $(\phi, \psi)$ such that $\phi \in \mathscr{X}_{\Lambda}(\boldsymbol{c}), \psi \in \mathscr{Y}_{\Lambda}(\boldsymbol{c})$ and $\psi \circ \phi=0$. If $\pi: \mathscr{L}_{\Lambda}(\boldsymbol{c}) \rightarrow \mathscr{X}_{\Lambda}(\boldsymbol{c})$ is the canonical projection, then $\pi$ is a vector bundle. Consequently, if $\mathscr{E}_{\Lambda}^{\prime}(\boldsymbol{c})$ is the set of pairs $(\phi, \psi) \in \mathscr{E}_{\Lambda}(\boldsymbol{c})$ such that $\psi$ is a surjection and $\pi^{\prime}$ is the restriction of $\pi$ to $\mathscr{E}_{\Lambda}^{\prime}(\boldsymbol{c})$, then $\pi^{\prime}$ is locally trivial (with fiber isomorphic to $\mathrm{GL}(\boldsymbol{c}))$. In particular, if $\mathscr{W}$ is an open subset of $\mathscr{L}_{\Lambda}^{\prime}(\boldsymbol{c})$, then $\pi^{\prime}(\mathscr{W})$ is an open subset of $\mathscr{X}_{\Lambda}(\boldsymbol{c})$.

There exists a regular map $\Theta: \mathscr{E}_{\Lambda}^{\prime}(\boldsymbol{c}) \rightarrow \bmod _{\Lambda}(\boldsymbol{c})$ such that $\Theta(\phi, \psi) \simeq \operatorname{Coker} \phi$ for all $(\phi, \psi) \in \mathscr{E}_{\Lambda}^{\prime}(\boldsymbol{c})$ (the proof is analogous to the proof of [Richmond 2001, Lemma 9], hence we omit it). Since $U=\pi\left(\Theta^{-1}(\mathscr{V})\right)$, the claim follows.

We will also need the following:

Lemma 4.4. Let $\Lambda$ be an algebra, $\boldsymbol{d}$ a dimension vector, $\mathscr{b}$ a $\mathrm{GL}(\boldsymbol{d})$-invariant irreducible closed subset of $\bmod _{\Lambda}(\boldsymbol{d})$, and $\boldsymbol{c}$ a dimension vector such that $\mathscr{P}_{\Lambda}(\boldsymbol{c}) \neq \varnothing$.

(1) If $\mathcal{U}$ is a nonempty open subset of $\mathscr{Q}_{\Lambda}(\boldsymbol{c})$, then

$$
\operatorname{span}\left\{c_{\mathscr{C}}^{\phi} \mid \phi \in \mathscr{U}\right\}=\operatorname{span}\left\{c_{\mathscr{C}}^{\phi} \mid \phi \in \mathscr{X}_{\Lambda}(c)\right\} .
$$

(2) If $\mathscr{V}$ is a nonempty open subset of $\mathscr{P}_{\Lambda}(\boldsymbol{c})$, then

$$
\operatorname{span}\left\{c_{\Lambda, \mathscr{C}}^{V} \mid V \in \mathscr{V}\right\}=\operatorname{span}\left\{c_{\Lambda, \mathscr{C}}^{V} \mid V \in \mathscr{P}_{\Lambda}(\boldsymbol{c})\right\} .
$$

Proof. Using Lemma 4.3, it is sufficient to prove the first assertion. Let $\phi_{1}, \ldots, \phi_{m} \in$ $\mathscr{X}_{\Lambda}(\boldsymbol{c})$ be such that $c_{\mathscr{C}}^{\phi_{1}}, \ldots, c_{\mathscr{C}}^{\phi_{m}}$ form a basis of $\operatorname{span}\left\{c_{\mathscr{C}}^{\phi} \mid \phi \in \mathscr{X}_{\Lambda}(\boldsymbol{c})\right\}$. There exist $M_{1}, \ldots, M_{m} \in \mathscr{C}$ such that

$$
\operatorname{det}\left[c_{\mathscr{C}}^{\phi_{i}}\left(M_{j}\right)\right]_{1 \leq i, j \leq m} \neq 0 .
$$

It suffices to show there exist $\psi_{1}, \ldots, \psi_{m} \in \mathcal{U}$ such that

$$
\operatorname{det}\left[c_{\mathscr{C}}^{\psi_{i}}\left(M_{j}\right)\right]_{1 \leq i, j \leq m} \neq 0 .
$$


However, the regular function

$$
\phi: \mathscr{X}_{\Lambda}(\boldsymbol{c})^{m} \rightarrow \mathbb{k}, \quad\left(\psi_{1}, \ldots, \psi_{m}\right) \mapsto \operatorname{det}\left[c_{\mathscr{C}}^{\psi_{i}}\left(M_{j}\right)\right]_{1 \leq i, j \leq m},
$$

is not a zero function, hence the claim follows.

Now we use the above construction to describe generating sets of semi-invariants. We present two such sets. Depending on the situation, it will be more convenient to use one of them.

Let $\Lambda$ be an algebra with Gabriel quiver $Q, \boldsymbol{d}$ a dimension vector, $\mathscr{C}$ an irreducible component of $\bmod _{\Lambda}(\boldsymbol{d})$, and $\theta$ a weight such that $\operatorname{SI}[\mathscr{C}]_{\theta} \neq 0$. There exists unique $\boldsymbol{c}_{\theta} \in \mathbb{Z}^{Q_{0}}$ such that $\theta=\left\langle\boldsymbol{c}_{\theta},-\right\rangle_{\mathbb{k} Q}$. Since $\operatorname{SI}\left[\mathrm{rep}_{Q}(\boldsymbol{d})\right]_{\theta} \neq 0$, we may assume that $\boldsymbol{c}_{\theta}$ is a dimension vector. We explain this more precisely.

If $\theta^{\prime}$ and $\theta^{\prime \prime}$ are weights, then $\operatorname{SI}\left[\operatorname{rep}_{Q}(\boldsymbol{d})\right]_{\theta^{\prime}}$ and $\operatorname{SI}\left[\operatorname{rep}_{Q}(\boldsymbol{d})\right]_{\theta^{\prime \prime}}$ are equal and both nonzero if and only if $\theta^{\prime}$ and $\theta^{\prime \prime}$ are $\boldsymbol{d}$-equivalent, i.e., $\theta^{\prime}(x)=\theta^{\prime \prime}(x)$ for all $x \in(\operatorname{supp} \boldsymbol{d})_{0}$. Now [Derksen and Weyman 2000, Theorem 1] (see also [Schofield and van den Bergh 2001, Theorem 2.3]) implies that there exists a dimension vector $\boldsymbol{c}$ such that the weights $\theta$ and $\langle\boldsymbol{c},-\rangle_{\mathbb{k} Q}$ are $\boldsymbol{d}$-equivalent. Consequently, we may assume that we only consider weights of this form.

It is clear that $\mathscr{P}_{\mathbb{k} Q}\left(\boldsymbol{c}_{\theta}\right) \neq \varnothing$ (the category rep $Q$ is hereditary); hence also $\mathscr{X}_{\mathbb{k} Q}\left(\boldsymbol{c}_{\theta}\right) \neq \varnothing$. It follows from [Chindris 2009, Corollary 2.5] that the semi-invariants $c_{\boldsymbol{d}}^{\phi}$, for $\phi \in \mathscr{X}_{\mathbb{k} Q}\left(\boldsymbol{c}_{\theta}\right)$, span $\operatorname{SI}\left[\operatorname{rep}_{Q}(\boldsymbol{d})\right]_{\theta}$. Since $\mathscr{C}$ is a closed GL $(\boldsymbol{d})$-invariant subset of $\operatorname{rep}_{Q}(\boldsymbol{d})$ and char $\mathbb{k}=0$, it follows that the semi-invariants $c_{\mathscr{C}}^{\phi}, \phi \in \mathscr{X}_{\mathbb{k} Q}\left(\boldsymbol{c}_{\theta}\right)$, span $\mathrm{SI}[\mathrm{G}]_{\theta}$.

We list some consequences:

Lemma 4.5. Let $\Lambda$ be an algebra, $\boldsymbol{d}$ a dimension vector, $\mathscr{C}$ an irreducible component of $\bmod _{\Lambda}(\boldsymbol{d})$, and $\theta$ a weight such that $\mathrm{SI}[\mathfrak{b}]_{\theta} \neq 0$. If $\mathscr{C}^{\prime}$ is a summand of $\mathscr{b}$, then $\operatorname{SI}\left[\mathscr{C}^{\prime}\right]_{\theta} \neq 0$.

Proof. Write $\mathscr{C}=\mathscr{C}^{\prime} \oplus \mathscr{C}^{\prime \prime}$. By assumption there exist $\phi \in \mathscr{X}_{\mathbb{k} Q}\left(\boldsymbol{c}_{\theta}\right)$ and $M \in \mathscr{C}$ such that $c_{\mathscr{C}}^{\phi}(M) \neq 0$. Without loss of generality we may assume that $M=M^{\prime} \oplus M^{\prime \prime}$ for $M^{\prime} \in \mathscr{C}^{\prime}$ and $M^{\prime \prime} \in \mathscr{C}^{\prime \prime}$. Lemma 4.1(1) implies that $\theta\left(\operatorname{dim} M_{1}\right)=0=\theta\left(\operatorname{dim} M_{2}\right)$. Consequently, Lemma 4.1(2) implies that $c_{\mathscr{C}}^{\phi}(M)=c_{\mathscr{C}^{\prime}}^{\phi}\left(M^{\prime}\right) \cdot c_{\mathscr{C}^{\prime \prime}}^{\phi}\left(M^{\prime \prime}\right)$. In particular, $c_{\mathscr{C}^{\prime}}^{\phi}\left(M^{\prime}\right) \neq 0$.

Lemma 4.6. Let $\Lambda$ be an algebra, $\boldsymbol{d}$ a dimension vector, $\mathscr{b}$ an irreducible component of $\bmod _{\Lambda}(\boldsymbol{d})$, and $\theta$ a weight such that $\mathrm{SI}\left[\mathscr{C}_{\theta} \neq 0\right.$. Then there exists an open subset $\mathscr{U}$ of $\mathscr{Q}_{\mathbb{k} Q}\left(\boldsymbol{c}_{\theta}\right)$ such that $c_{\mathscr{C}}^{\phi} \neq 0$ for all $\phi \in \mathcal{U}$.

Proof. There exist $\phi_{0} \in \mathscr{X}_{\mathbb{k} Q}\left(\boldsymbol{c}_{\theta}\right)$ and $M \in \mathscr{C}$ such that $c_{\mathscr{C}}^{\phi_{0}}(M) \neq 0$. We define a function $\Phi: \mathscr{Q}_{\mathbb{k} Q}\left(\boldsymbol{c}_{\theta}\right) \rightarrow \mathbb{k}$ by

$$
\Phi(\phi):=c_{\mathscr{C}}^{\phi}(M) \quad\left(\phi \in \mathscr{X}_{\mathbb{k} Q}\left(\boldsymbol{c}_{\theta}\right)\right) .
$$


This is a regular function and we take $U:=\Phi^{-1}\left(\mathbb{k}^{\times}\right)$.

Now we present the second construction. As above let $\Lambda$ be an algebra with Gabriel quiver $Q, \boldsymbol{d}$ a dimension vector, $\mathscr{C}$ an irreducible component of $\bmod _{\Lambda}(\boldsymbol{d})$, and $\theta$ a weight such that SI[ $\mathscr{C}]_{\theta} \neq 0$. Next, let $\Lambda_{\mathscr{C}}:=\Lambda / \operatorname{Ann} \mathscr{C}$, where Ann $\mathscr{C}:=$ $\bigcap_{M \in \mathscr{C}}$ Ann $M$. Consequently, $\mathscr{C}$ is a faithful irreducible component of $\bmod _{\Lambda_{\mathscr{C}}}(\boldsymbol{d})$. Again there exists $\boldsymbol{c}_{\theta, \mathscr{C}} \in \mathbb{Z}^{Q_{0}}$ such that $\theta=\left\langle\boldsymbol{c}_{\theta, \mathscr{C}},-\right\rangle_{\Lambda_{\mathscr{C}}}$. Since $\mathrm{SI}[\mathscr{C}]_{\theta} \neq 0$, $\boldsymbol{c}_{\theta, \mathscr{C}}$ is a dimension vector and $\mathscr{P}_{\mathscr{C}}(\theta):=\mathscr{P}_{\Lambda_{\mathscr{C}}}\left(\boldsymbol{c}_{\theta, \mathscr{C}}\right) \neq \varnothing$ (see [Derksen and Weyman 2002, Theorem 1]). Moreover, [Derksen and Weyman 2002, Theorem 1] also says that $\mathrm{SI}[\mathscr{C}]_{\theta}$ is spanned by the semi-invariants $c_{\Lambda_{\mathscr{C}}, \mathscr{C}}^{V}, V \in \mathscr{P}_{\mathscr{C}}(\theta)$.

It is known that $\overline{\mathscr{P}}_{\mathscr{C}}(\theta)$ is an irreducible component of $\bmod _{\Lambda_{\mathscr{C}}}\left(\boldsymbol{c}_{\theta, \mathscr{C}}\right)$ [Barot and Schröer 2001, Proposition 3.1]. It is quite easy to observe that the generic decomposition of $\overline{\mathscr{P}}_{\mathscr{C}}(\theta)$ is of the form

$$
\overline{\mathscr{P}}_{\mathscr{C}}(\theta)=\overline{\mathscr{P}}_{\Lambda_{\complement}}\left(\boldsymbol{c}_{1}\right) \oplus \cdots \oplus \overline{\mathscr{P}}_{\Lambda_{\complement}}\left(\boldsymbol{c}_{n}\right)
$$

for some dimension vectors $\boldsymbol{c}_{1}, \ldots, \boldsymbol{c}_{n}$ such that $\boldsymbol{c}_{\theta, \mathscr{C}}=\boldsymbol{c}_{1}+\cdots+\boldsymbol{c}_{n}$. Obviously $\boldsymbol{c}_{1}, \ldots, \boldsymbol{c}_{n}$ are the generic summands of $\boldsymbol{c}_{\theta, \mathscr{C}}\left(\right.$ at $\left.\overline{\mathscr{P}}_{\mathscr{C}}(\theta)\right)$. If we put $\theta_{i}:=\left\langle\boldsymbol{c}_{i},-\right\rangle_{\Lambda_{\mathscr{C}}}$, then we call the presentation

$$
\theta=\theta_{1}+\cdots+\theta_{n}
$$

the generic decomposition of $\theta$ at $\mathscr{C}$.

As a first consequence we get the following:

Lemma 4.7. Let $\Lambda$ be an algebra, $\boldsymbol{d}$ a dimension vector, $\mathscr{b}$ an irreducible component of $\bmod _{\Lambda}(\boldsymbol{d})$, and $\theta$ a weight such that $\operatorname{SI}[\mathscr{C}]_{\theta} \neq 0$. If $\theta=\theta_{1}+\cdots+\theta_{n}$ is the generic decomposition of $\theta$ at $\mathscr{C}$, then the image of the map

$$
\mathrm{SI}[\mathscr{C}]_{\theta_{1}} \times \cdots \times \mathrm{SI}[\mathscr{C}]_{\theta_{n}} \rightarrow \operatorname{SI}[\mathscr{C}]_{\theta}, \quad\left(f_{1}, \ldots, f_{n}\right) \mapsto f_{1} \cdots f_{n},
$$

spans $\mathrm{SI}[\mathscr{C}]_{\theta}$. In particular, $\mathrm{SI}[\mathscr{C}]_{\theta_{i}} \neq 0$ for each $i \in[1, n]$.

Proof. Let $\boldsymbol{c}_{1}, \ldots, \boldsymbol{c}_{n}$ be the dimension vectors corresponding to the weights $\theta_{1}, \ldots, \theta_{n}$, respectively, in the sense explained above. The set $\mathscr{V}$ of $V \in \mathscr{P}_{\mathscr{C}}(\theta)$ such that $V \simeq V_{1} \oplus \cdots \oplus V_{n}$ for $V_{1} \in \mathscr{P}_{\Lambda_{\mathscr{\zeta}}}\left(\boldsymbol{c}_{1}\right), \ldots, V_{n} \in \mathscr{P}_{\Lambda_{\mathscr{\zeta}}}\left(\boldsymbol{c}_{n}\right)$ contains an open subset of $\mathscr{P}_{\mathscr{C}}(\theta)$. Lemma 4.4(2) implies that $\mathrm{SI}[\mathscr{C}]_{\theta}$ is spanned by the semi-invariants $c_{\Lambda_{\mathscr{\ell}}, \mathscr{G}}^{V_{1} \oplus \cdots V_{n}}, V_{1} \in \mathscr{P}_{\Lambda_{\mathscr{\ell}}}\left(\boldsymbol{c}_{1}\right), \ldots, V_{n} \in \mathscr{P}_{\Lambda_{\mathscr{G}}}\left(\boldsymbol{c}_{n}\right)$. Moreover, Lemma 4.2 implies that

$$
c_{\Lambda_{\mathscr{C}, \mathscr{C}}}^{V_{1} \oplus \cdots V_{n}}=c_{\Lambda_{\mathscr{C}, \mathscr{C}}}^{V_{1}} \cdots c_{\Lambda_{\mathscr{C}, \mathscr{C}}}^{V_{n}}
$$

for all $V_{1} \in \mathscr{P}_{\Lambda_{\mathscr{\zeta}}}\left(\boldsymbol{c}_{1}\right), \ldots, V_{n} \in \mathscr{P}_{\Lambda_{\mathscr{\zeta}}}\left(\boldsymbol{c}_{n}\right)$, hence the claim follows.

As a next consequence we obtain the following useful fact: 
Lemma 4.8. Let $\Lambda$ be an algebra, $\boldsymbol{d}$ a dimension vector, $\mathscr{b}$ an irreducible component of $\bmod _{\Lambda}(\boldsymbol{d})$, and $\theta$ a weight such that $\mathrm{SI}\left[{ }^{\mathfrak{C}}\right]_{\theta} \neq 0$. If $\mathscr{P}_{\mathscr{C}}(\theta)$ contains a dense orbit, then $\operatorname{dim}_{\mathbb{k}} \operatorname{SI}[\mathscr{C}]_{\theta}=1$.

Proof. If $\mathcal{O}(V)$ is a dense orbit in $\mathscr{P}_{\mathscr{C}}(\theta)$, then Lemma 4.4(2) implies that $\mathrm{SI}_{[}[]_{\theta}$ is spanned by the semi-invariant $c_{\Lambda_{\ell}, \mathscr{\ell}}^{V}$, hence the claim follows.

Consequently, we get the following:

Corollary 4.9. Let $\Lambda$ be an algebra, $\boldsymbol{d}$ a dimension vector, $\mathscr{C}$ an irreducible component of $\bmod _{\Lambda}(\boldsymbol{d})$, and $\theta$ a weight such that $\mathrm{SI}\left[{ }^{\mathfrak{C}}\right]_{\theta} \neq 0$. If $\boldsymbol{c}^{\prime}$ is a generic summand of $\boldsymbol{c}_{\theta, \mathscr{C}}$ at $\overline{\mathscr{P}}_{\mathscr{C}}(\theta)$ such that $\mathscr{P}_{\Lambda_{\mathscr{C}}}\left(\boldsymbol{c}^{\prime}\right)$ contains a dense orbit, then

$$
\mathrm{SI}[\mathscr{C}]_{\theta} \simeq \operatorname{SI}[\mathscr{C}]_{\theta-\theta^{\prime}},
$$

where $\theta^{\prime}:=\left\langle\boldsymbol{c}^{\prime},-\right\rangle_{\Lambda_{\varphi}}$.

Proof. Lemma 4.7 implies that SI[ $\mathscr{C}]_{\theta^{\prime}} \neq 0$. Together with Lemma 4.8 this implies that $\operatorname{dim}_{\mathbb{k}} \mathrm{SI}[\mathscr{C}]_{\theta^{\prime}}=1$. Fix a nonzero semi-invariant $f \in \mathrm{SI}[\mathscr{C}]_{\theta^{\prime}}$. Lemma 4.7 implies that the map

$$
\mathrm{SI}[\mathscr{C}]_{\theta-\theta^{\prime}} \rightarrow \mathrm{SI}[\mathscr{C}]_{\theta}, \quad c \mapsto f \cdot c,
$$

is surjective. Since $\mathscr{C}$ is irreducible, this is also injective, and the claim follows.

\section{Moduli spaces}

Let $\Lambda$ be an algebra, $\boldsymbol{d}$ a dimension vector, and $\mathscr{C}$ an irreducible component of $\bmod _{\Lambda}(\boldsymbol{d})$. If $\theta$ is a weight, then a $\Lambda$-module $M \in \mathscr{C}$ is called $\theta$-semistable if there exists $f \in \operatorname{SI}[\mathscr{C}]_{p \theta}$, for some $p \in \mathbb{N}_{+}$, such that $f(M) \neq 0$. King [1994] has proved that $M$ is $\theta$-semistable if and only if $\theta(\operatorname{dim} M)=0$ and $\theta(\operatorname{dim} N) \leq 0$ for each submodule $N$ of $M$. We denote by $\mathscr{C}_{\theta}^{\text {ss }}$ the set of $\theta$-semistable $\Lambda$-modules in $\mathscr{C}$. King has also constructed a coarse moduli $\mathcal{M ( \mathscr { C }})_{\theta}^{\text {ss }}$ for the $\theta$-semistable modules in $\mathscr{C}$ (up to an equivalence, which identifies modules which have the same simple composition factors within the category of $\theta$-semistable modules). By definition,

$$
\mu(\mathfrak{b})_{\theta}^{\mathrm{ss}}=\operatorname{Proj}\left(\bigoplus_{p \in \mathbb{N}} \operatorname{SI}[\mathfrak{b}]_{p \theta}\right) .
$$

Lemma 5.1. Let $\Lambda$ be an algebra, $\boldsymbol{d}$ a dimension vector, $\mathscr{b}$ an irreducible component of $\bmod _{\Lambda}(\boldsymbol{d})$, and $\theta$ a weight such that $\mathscr{C}_{\theta}^{\mathrm{ss}} \neq \varnothing$. If $\mathscr{C}=\mathscr{C}_{1} \oplus \mathscr{C}_{2}$ for irreducible components $\mathscr{C}_{1}$ and $\mathscr{C}_{2}$ of $\bmod _{\Lambda}\left(\boldsymbol{d}_{1}\right)$ and $\bmod _{\Lambda}\left(\boldsymbol{d}_{2}\right)$, respectively, and $\mathscr{C}_{2}$ is an orbit closure, then

$$
\mu(\mathscr{b})_{\theta}^{\mathrm{ss}} \simeq M\left(\mathscr{b}_{1}\right)_{\theta}^{\mathrm{ss}} .
$$


Proof. Without loss of generality we may assume that $\mathrm{SI}[\mathscr{C}]_{\theta} \neq 0$. Then we will show that

$$
\mathrm{SI}[\mathscr{C}]_{p \theta} \simeq \mathrm{SI}\left[\mathscr{C}_{1}\right]_{p \theta}
$$

for each $p \in \mathbb{N}$.

Let $\mathscr{C}_{2}=\overline{\mathscr{O}(M)}$. Consider the map $\Phi: \mathscr{C}_{1} \rightarrow \mathscr{C}$ given by

$$
\Phi(N):=N \oplus M \quad\left(N \in \mathscr{C}_{1}\right) .
$$

We will show that $\Phi^{*}: \mathbb{k}[\mathscr{C}] \rightarrow \mathbb{k}\left[\mathfrak{C}_{1}\right]$ induces an isomorphism $\Phi_{p}^{*}: \operatorname{SI}\left[\mathfrak{C}_{p \theta} \simeq\right.$ $\mathrm{SI}\left[\mathscr{C}_{1}\right]_{p \theta}$ for each $p \in \mathbb{N}$.

Fix $p \in \mathbb{N}$. Since $\operatorname{GL}(\boldsymbol{d}) \times\left(\mathscr{C}_{1} \oplus\{M\}\right)$ is a dense subset of $\mathscr{C}$, it is clear that $\Phi_{p}^{*}$ is a monomorphism. Thus it remains to show that $\Phi_{p}^{*}$ is an epimorphism. Let $\mathscr{X}:=\mathscr{X}_{\mathbb{k} Q}(p \theta)$, where $Q$ is the Gabriel quiver of $\Lambda$. Using Lemma 4.4(1), it suffices to show that there exists an open subset $\mathscr{U}$ of $\mathscr{L}$ such that $c_{\mathscr{C}_{1}}^{\phi}$ is in the image of $\Phi_{p}^{*}$ for each $\phi \in \mathcal{U}$.

It follows from Lemma 4.5 that $\mathrm{SI}\left[\mathscr{C}_{2}\right]_{p \theta} \neq 0$. Using Lemma 4.6, we obtain that there exists an open subset $\mathscr{U}$ of $\mathscr{L}$ such that $c_{\mathscr{C}_{2}}^{\phi} \neq 0$ for each $\phi \in \mathscr{U}$. In particular, $c_{\mathscr{C}_{2}}^{\phi}(M) \neq 0$ for each $\phi \in \mathcal{U}$. Now it follows from Lemma 4.1 that

$$
c_{\mathscr{C}_{1}}^{\phi}=\Phi_{p}^{*}\left(\frac{1}{c_{\mathscr{C}_{2}}^{\phi}(M)} c_{\mathscr{C}}^{\phi}\right)
$$

for each $\phi \in U$.

\section{Moduli spaces for the tame quasitilted algebras}

The aim of this section is to prove Theorem 2. Let $\Lambda$ be a tame quasitilted algebra, $\boldsymbol{d}$ a dimension vector, $\mathscr{C}$ an irreducible component of $\bmod _{\Lambda}(\boldsymbol{d})$, and $\theta$ a weight such that $\mathscr{C}_{\theta}^{\text {ss }} \neq \varnothing$. We show that $M(\mathscr{C})_{\theta}^{\text {ss }}$ is a product of projective spaces. Let $\Lambda^{\prime}:=\Lambda / \operatorname{Ann} \mathscr{C}$.

We know that there exist Schur roots $\boldsymbol{d}_{1}, \ldots, \boldsymbol{d}_{n}$ such that

$$
\mathscr{C}=\mathscr{C}_{1} \oplus \cdots \oplus \mathscr{C}_{n},
$$

where $\mathscr{C}_{i}:=\mathscr{C}\left(\boldsymbol{d}_{i}\right), i \in[1, n]$. Using Lemma 5.1 , we may assume that $\boldsymbol{d}_{1}, \ldots, \boldsymbol{d}_{n}$ are isotropic.

Now let $\boldsymbol{c}_{1}, \ldots, \boldsymbol{c}_{m}$ be the generic summands of $\boldsymbol{c}_{\theta, \mathscr{C}}$ at $\overline{\mathscr{P}}_{\Lambda^{\prime}}\left(\boldsymbol{c}_{\theta, \mathscr{C}}\right)$. Using Corollary 4.9, we may assume that for each $j \in[1, m], \mathscr{P}_{\Lambda^{\prime}}\left(\boldsymbol{c}_{j}\right)$ does not contain a dense orbit. Since $\overline{\mathscr{P}}_{\Lambda^{\prime}}\left(\boldsymbol{c}_{j}\right)$ is an indecomposable irreducible component, this implies that there exist infinitely many indecomposable $\Lambda^{\prime}$-modules of dimension vector $\boldsymbol{c}_{j}$, for each $j \in[1, m]$. This also means that, for each $j \in[1, m]$, there exist infinitely many indecomposable $\Lambda$-modules of dimension vector $\boldsymbol{c}_{j}$, hence 
$\boldsymbol{c}_{1}, \ldots, \boldsymbol{c}_{m}$ are isotropic roots of $\chi_{\Lambda}$ (using more detailed knowledge of $\bmod \Lambda$ one could also show that they are Schur roots, but we will not use this).

Before we formulate the next lemma let us recall that if $i \in[1, n], j \in[1, m]$, $M \in \mathscr{C}_{i}$ and $V \in \mathscr{P}_{\Lambda^{\prime}}\left(\boldsymbol{c}_{j}\right)$, then $c_{\mathscr{C}_{i}}^{V}(M)=0$ if and only if $\operatorname{Hom}_{\Lambda}(V, M) \neq 0$ or $\operatorname{Hom}_{\Lambda}\left(M, \tau_{\Lambda^{\prime}} V\right) \neq 0$.

Lemma 6.1. In the above notation, either $\boldsymbol{c}_{j}$ is a multiplicity of $\boldsymbol{d}_{i}$ or the intersection supp $\boldsymbol{d}_{i} \cap \operatorname{supp} \boldsymbol{c}_{j}$ is empty for all $i \in[1, n]$ and $j \in[1, m]$.

Proof. Fix $i \in[1, n]$ and $j \in[1, m]$. Note that Lemmas 4.5 and 4.7 imply that $\operatorname{SI}\left[\mathscr{C}_{i}\right]_{\theta^{\prime}} \neq 0$, where $\theta^{\prime}:=\left\langle\boldsymbol{c}_{j},-\right\rangle_{\Lambda^{\prime}}$. Assume that neither $\boldsymbol{c}_{j}$ is a multiplicity of $\boldsymbol{d}_{i}$ nor supp $\boldsymbol{d}_{i} \cap \operatorname{supp} \boldsymbol{c}_{j}=\varnothing$. Then Section 2 implies that one of the following holds:

(1) $\operatorname{Hom}_{\Lambda}(V, M) \neq 0$ for each indecomposable $\Lambda$-module $M$ with dimension vector $\boldsymbol{d}_{i}$ and each indecomposable $\Lambda$-module $V$ with dimension vector $\boldsymbol{c}_{j}$.

(2) $\operatorname{Hom}_{\Lambda}(M, V) \neq 0$ for each indecomposable $\Lambda$-module $M$ with dimension vector $\boldsymbol{d}_{i}$ and each indecomposable $\Lambda$-module $V$ with dimension vector $\boldsymbol{c}_{j}$.

In the first case we immediately obtain that $\mathrm{SI}\left[\mathscr{C}_{i}\right]_{\theta^{\prime}}=0$, a contradiction. We show that we get the same conclusion in the second case.

Since $\Lambda$, hence also $\Lambda^{\prime}$, are tame and there are infinitely many indecomposable $\Lambda^{\prime}$-modules in $\mathscr{P}_{\Lambda^{\prime}}\left(\boldsymbol{c}_{j}\right)$, [Crawley-Boevey 1988, Theorem D] implies that there is a nonempty open subset $\mathscr{V}$ of $\mathscr{P}_{\Lambda^{\prime}}\left(\boldsymbol{c}_{j}\right)$ such that $\tau_{\Lambda^{\prime}} V \simeq V$ for each $V \in \mathscr{V}$. In particular, $\operatorname{dim} \tau_{\Lambda^{\prime}} V=\boldsymbol{c}_{j}$ for each $V \in \mathscr{V}$. Thus (2) together with Lemma 4.4(2) implies that $\mathrm{SI}\left[\mathscr{C}_{i}\right]_{\theta^{\prime}}=0$, and this finishes the proof.

Let $I^{\prime}$ be the set of $i \in[1, n]$ such that there exists $j \in[1, m]$ with $\boldsymbol{c}_{j}$ a multiplicity of $\boldsymbol{d}_{i}$. Let $I^{\prime \prime}:=[1, n] \backslash I$. Lemma 6.1 implies that supp $\boldsymbol{d}_{p} \cap \operatorname{supp} \boldsymbol{d}_{q}=\varnothing$ if $p \in I^{\prime}$ and $q \in I^{\prime \prime}\left(\right.$ since supp $\boldsymbol{d}_{p}=\operatorname{supp} \boldsymbol{c}_{j}$ for some $\left.j \in J\right)$. Thus $\mathscr{C}_{=}=\mathscr{C}_{1} \times \mathscr{C}_{2}$, where

$$
\mathscr{C}_{1}:=\bigoplus_{i \in I^{\prime}} \mathscr{C}\left(\boldsymbol{d}_{i}\right) \quad \text { and } \quad \mathscr{C}_{2}:=\bigoplus_{i \in I^{\prime \prime}} \mathscr{C}\left(\boldsymbol{d}_{i}\right)
$$

Consequently,

$$
\mu(\mathscr{b})_{\theta}^{\mathrm{ss}}=\mu\left(\mathscr{b}_{1}\right)_{\theta}^{\mathrm{ss}} \times M\left(\mathscr{C}_{2}\right)_{\theta}^{\mathrm{ss}} .
$$

If $I^{\prime} \neq[1, n] \neq I^{\prime \prime}$, then we get Theorem 2 by induction. Hence we have only two cases to consider: either $I^{\prime}=[1, n]$ or $I^{\prime \prime}=[1, n]$.

First assume that $I^{\prime \prime}=[1, n]$. Since $\mathscr{C}$ is a faithful component over $\Lambda^{\prime}, \boldsymbol{d}$ is a sincere dimension vector over $\Lambda^{\prime}$. On the other hand, supp $\boldsymbol{c}_{j} \cap \operatorname{supp} \boldsymbol{d}=\varnothing$, hence $\boldsymbol{c}_{j}=0$. Consequently, $\theta=0$. Thus

$$
\mathrm{SI}[\mathscr{C}]_{p \theta}=\mathbb{k}[\mathscr{C}]^{\mathrm{GL}(\boldsymbol{d})}=\mathbb{k}
$$

for each $p \in \mathbb{N}$, and

$$
M(\mathscr{C})_{\theta}^{\mathrm{ss}}=\operatorname{Proj}(\mathbb{k}[T])=\{*\} .
$$


Now assume that $I^{\prime}=[1, n]$. We can make another reduction in this case. Let $\boldsymbol{d}_{1}^{\prime}, \ldots, \boldsymbol{d}_{l}^{\prime}$ be the pairwise different vectors among $\boldsymbol{d}_{1}, \ldots, \boldsymbol{d}_{n}$. For each $p \in[1, l]$, let $I_{p}$ be the set of $i \in[1, n]$ such that $\boldsymbol{d}_{i}=\boldsymbol{d}_{p}^{\prime}$. Let $\mathscr{C}_{p}^{\prime}:=\bigoplus_{i \in I_{p}} \mathscr{C}\left(\boldsymbol{d}_{i}\right)$ for $p \in[1, l]$. Lemma 6.1 again implies that $\operatorname{supp} \boldsymbol{d}_{p}^{\prime} \cap \operatorname{supp} \boldsymbol{d}_{q}^{\prime}=\varnothing$ if $p, q \in[1, l]$ and $p \neq q$. Consequently,

$$
\mathscr{C}=\mathscr{C}_{1}^{\prime} \times \cdots \times \mathscr{C}_{l}^{\prime}
$$

and

$$
M(\mathscr{C})_{\theta}^{\mathrm{ss}}=M\left(\mathscr{C}_{1}^{\prime}\right)_{\theta}^{\mathrm{ss}} \times \cdots \times M\left(\mathscr{C}_{l}^{\prime}\right)_{\theta}^{\mathrm{ss}} .
$$

If $l>1$, then Theorem 2 follows by induction again, thus we may assume $l=1$.

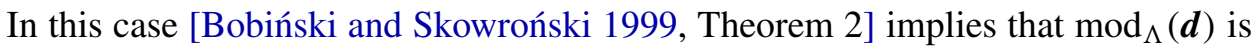
irreducible, hence $\mathscr{b}=\bmod _{\Lambda}(\boldsymbol{d})$. Thus Theorem 2 is a result of the following:

Proposition 6.2. Let $\Lambda$ be a tame quasitilted algebra and let $\boldsymbol{h}$ be an isotropic Schur root of $\chi_{\Lambda}$. If $n, p \in \mathbb{N}_{+}$, then

$$
\mathcal{M}\left(\bmod _{\Lambda}(n \boldsymbol{h})\right)_{p\langle\boldsymbol{h},-\rangle_{\Lambda}}^{\mathrm{ss}} \simeq \mathbb{P}_{\mathbb{k}}^{n} .
$$

Proof. This is a part of [Domokos and Lenzing 2002, Theorem 7.1]. One may also give a more direct proof, using a description of the semi-invariants for concealedcanonical algebras (the support of $\boldsymbol{h}$ is a concealed-canonical algebra) which implies that $\bigoplus_{q \in \mathbb{N}} \operatorname{SI}\left[\bmod _{\Lambda}(n \boldsymbol{h})\right]_{q\langle\boldsymbol{h},-\rangle_{\Lambda}}$ is the polynomial ring in $n+1$ variables (see [Bobiński 2015, Proposition 6.2]).

\section{Acknowledgements}

The paper was written during the author's stay at the Bielefeld University, which was supported by CRC 701. The author also acknowledges the support of National Science Center grant no. 2011/03/B/ST1/00847.

\section{References}

[Assem et al. 2006] I. Assem, D. Simson, and A. Skowroński, Elements of the representation theory of associative algebras, Vol. 1: Techniques of representation theory, London Mathematical Society Student Texts 65, Cambridge University Press, Cambridge, 2006. MR 2006j:16020 Zbl 1092.16001

[Auslander et al. 1997] M. Auslander, I. Reiten, and S. O., Representation theory of Artin algebras, Cambridge Studies in Advanced Mathematics 36, Cambridge University Press, Cambridge, 1997. MR 98e:16011

[Barot and Schröer 2001] M. Barot and J. Schröer, "Module varieties over canonical algebras", J. Algebra 246: 1 (2001), 175-192. MR 2003e:16013 Zbl 1036.16010

[Bobiński 2015] G. Bobiński, "Semi-invariants for concealed-canonical algebras", J. Pure Appl. Algebra 219:1 (2015), 59-76. MR 3240823 Zbl 06334325

[Bobiński and Skowroński 1999] G. Bobiński and A. Skowroński, "Geometry of modules over tame quasi-tilted algebras”, Colloq. Math. 79:1 (1999), 85-118. MR 2000i:14067 Zbl 0994.16009 
[Carroll and Chindris 2012] A. T. Carroll and C. Chindris, "On the invariant theory for acyclic gentle algebras", preprint, 2012. arXiv 1210.3579

[Chindris 2009] C. Chindris, "Orbit semigroups and the representation type of quivers", J. Pure Appl. Algebra 213:7 (2009), 1418-1429. MR 2010a:16024 Zbl 1207.16012

[Chindris 2011] C. Chindris, "Geometric characterizations of the representation type of hereditary algebras and of canonical algebras", Adv. Math. 228:3 (2011), 1405-1434. MR 2012h:16033 Zbl 1252.16014

[Chindris 2013] C. Chindris, "On the invariant theory for tame tilted algebras", Algebra Number Theory 7:1 (2013), 193-214. MR 3037894 Zbl 06167117

[Crawley-Boevey 1988] W. W. Crawley-Boevey, "On tame algebras and bocses", Proc. London Math. Soc. (3) 56:3 (1988), 451-483. MR 89c:16028 Zbl 0661.16026

[Crawley-Boevey and Schröer 2002] W. Crawley-Boevey and J. Schröer, "Irreducible components of varieties of modules", J. Reine Angew. Math. 553 (2002), 201-220. MR 2004a:16020 Zbl 1062.16019

[Derksen and Weyman 2000] H. Derksen and J. Weyman, "Semi-invariants of quivers and saturation for Littlewood-Richardson coefficients”, J. Amer. Math. Soc. 13:3 (2000), 467-479. MR 2001g:16031 Zbl 0993.16011

[Derksen and Weyman 2002] H. Derksen and J. Weyman, "Semi-invariants for quivers with relations", J. Algebra 258:1 (2002), 216-227. MR 2003m:16018 Zbl 1048.16005

[Domokos 2002] M. Domokos, "Relative invariants for representations of finite dimensional algebras", Manuscripta Math. 108:1 (2002), 123-133. MR 2003d:16017 Zbl 1031.16014

[Domokos 2011] M. Domokos, “On singularities of quiver moduli”, Glasg. Math. J. 53:1 (2011), 131-139. MR 2012a:16029 Zbl 1241.16010

[Domokos and Lenzing 2002] M. Domokos and H. Lenzing, "Moduli spaces for representations of concealed-canonical algebras", J. Algebra 251:1 (2002), 371-394. MR 2003d:16016 Zbl 1013.16006

[Drozd 1980] J. A. Drozd, "Tame and wild matrix problems", pp. 242-258 in Representation theory,

II: Proceedings of the Second International Conference on Representations of Algebras (Ottawa, 1979), edited by V. Dlab and P. Gabriel, Lecture Notes in Math. 832, Springer, Berlin, 1980. MR 83b:16024 Zbl 0457.16018

[Happel 2001] D. Happel, "A characterization of hereditary categories with tilting object", Invent. Math. 144:2 (2001), 381-398. MR 2002a:18014 Zbl 1015.18006

[Happel et al. 1996] D. Happel, I. Reiten, and S. O., Tilting in abelian categories and quasitilted algebras, Memoirs of the American Mathematical Society 575, Amer. Math. Soc., Providence, RI, 1996. MR 97j:16009 Zbl 0849.16011

[Kerner 1989] O. Kerner, “Tilting wild algebras”, J. London Math. Soc. (2) 39:1 (1989), 29-47. MR 90d:16025 Zbl 0675.16013

[King 1994] A. D. King, "Moduli of representations of finite-dimensional algebras", Quart. J. Math. Oxford Ser. (2) 45:180 (1994), 515-530. MR 96a:16009 Zbl 0837.16005

[Lenzing and Skowroński 1996] H. Lenzing and A. Skowroński, "Quasi-tilted algebras of canonical type”, Colloq. Math. 71:2 (1996), 161-181. MR 97j:16019 Zbl 0870.16007

[Meltzer 1996] H. Meltzer, "Auslander-Reiten components for concealed-canonical algebras", Colloq. Math. 71:2 (1996), 183-202. MR 98b:16014 Zbl 0923.16016

[de la Peña 1991] J. A. de la Peña, "On the dimension of the module-varieties of tame and wild algebras”, Comm. Algebra 19:6 (1991), 1795-1807. MR 92i:16016 Zbl 0818.16013 
[Richmond 2001] N. J. Richmond, "A stratification for varieties of modules", Bull. London Math. Soc. 33:5 (2001), 565-577. MR 2002d:16017 Zbl 1054.16007

[Ringel 1984] C. M. Ringel, Tame algebras and integral quadratic forms, Lecture Notes in Mathematics 1099, Springer, Berlin, 1984. MR 87f:16027 Zbl 0546.16013

[Schofield 1991] A. Schofield, "Semi-invariants of quivers", J. London Math. Soc. (2) 43:3 (1991), 385-395. MR 92g:16019 Zbl 0779.16005

[Schofield and van den Bergh 2001] A. Schofield and M. van den Bergh, "Semi-invariants of quivers for arbitrary dimension vectors", Indag. Math. (N.S.) 12:1 (2001), 125-138. MR 2003e:16016 Zbl 1004.16012

[Skowroński 1998] A. Skowroński, “Tame quasi-tilted algebras”, J. Algebra 203:2 (1998), 470-490. MR 99b:16019 Zbl 0908.16013

[Skowroński 2006] A. Skowroński, "Selfinjective algebras: finite and tame type", pp. 169-238 in Trends in representation theory of algebras and related topics (Querétaro, 2004), edited by J. A. de la Peña and R. Bautista, Contemp. Math. 406, Amer. Math. Soc., Providence, RI, 2006. MR 2007f:16045 Zbl 1129.16013

[Skowroński and Weyman 2000] A. Skowroński and J. Weyman, "The algebras of semi-invariants of quivers”, Transform. Groups 5:4 (2000), 361-402. MR 2001m:16017 Zbl 0986.16004

[Skowroński and Zwara 1998] A. Skowroński and G. Zwara, "Degenerations for indecomposable modules and tame algebras", Ann. Sci. École Norm. Sup. (4) 31:2 (1998), 153-180. MR 99k:16032 Zbl 0915.16011

Communicated by David Benson

Received 2013-12-19 Revised 2014-02-14 Accepted 2014-06-14

gregbob@mat.umk.pl Faculty of Mathematics and Computer Science,

Nicolaus Copernicus University, Ulica Chopina 12/18,

87-100 Toruń, Poland 


\section{Algebra \& Number Theory}

msp.org/ant

\section{EDITORS}

MANAGING EDITOR

Bjorn Poonen

Massachusetts Institute of Technology

Cambridge, USA

\author{
EDITORIAL BOARD CHAIR \\ David Eisenbud \\ University of California \\ Berkeley, USA
}

BOARD OF EDITORS

Georgia Benkart

Dave Benson

Richard E. Borcherds

John H. Coates

J-L. Colliot-Thélène

Brian D. Conrad

Hélène Esnault

Hubert Flenner

Edward Frenkel

Andrew Granville

Joseph Gubeladze

Roger Heath-Brown

Craig Huneke

Yujiro Kawamata

János Kollár

Yuri Manin

Barry Mazur

Philippe Michel

Susan Montgomery
University of Wisconsin, Madison, USA

University of Aberdeen, Scotland

University of California, Berkeley, USA

University of Cambridge, UK

CNRS, Université Paris-Sud, France

University of Michigan, USA

Freie Universität Berlin, Germany

Ruhr-Universität, Germany

University of California, Berkeley, USA

Université de Montréal, Canada

San Francisco State University, USA

Oxford University, UK

University of Virginia, USA

University of Tokyo, Japan

Princeton University, USA

Northwestern University, USA

Harvard University, USA

École Polytechnique Fédérale de Lausanne

University of Southern California, USA
Shigefumi Mori

Raman Parimala

Jonathan Pila

Anand Pillay

Victor Reiner

Peter Sarnak

Joseph H. Silverman

Michael Singer

Vasudevan Srinivas

J. Toby Stafford

Bernd Sturmfels

Richard Taylor

Ravi Vakil

Michel van den Bergh

Marie-France Vignéras

Kei-Ichi Watanabe

Efim Zelmanov

Shou-Wu Zhang
RIMS, Kyoto University, Japan

Emory University, USA

University of Oxford, UK

University of Notre Dame, USA

University of Minnesota, USA

Princeton University, USA

Brown University, USA

North Carolina State University, USA

Tata Inst. of Fund. Research, India

University of Michigan, USA

University of California, Berkeley, USA

Harvard University, USA

Stanford University, USA

Hasselt University, Belgium

Université Paris VII, France

Nihon University, Japan

University of California, San Diego, USA

Princeton University, USA

\section{PRODUCTION}

production@msp.org

Silvio Levy, Scientific Editor

See inside back cover or msp.org/ant for submission instructions.

The subscription price for 2014 is US $\$ 225 /$ year for the electronic version, and $\$ 400 /$ year ( $\$ 55$, if shipping outside the US) for print and electronic. Subscriptions, requests for back issues and changes of subscribers address should be sent to MSP.

Algebra \& Number Theory (ISSN 1944-7833 electronic, 1937-0652 printed) at Mathematical Sciences Publishers, 798 Evans Hall \#3840, c/o University of California, Berkeley, CA 94720-3840 is published continuously online. Periodical rate postage paid at Berkeley, CA 94704, and additional mailing offices.

ANT peer review and production are managed by EditFLOW ${ }^{\circledR}$ from MSP.

\section{PUBLISHED BY}

- mathematical sciences publishers

nonprofit scientific publishing

http://msp.org/

(C) 2014 Mathematical Sciences Publishers 


\section{Algebra \& Number Theory}

Volume $8 \quad$ No. $6 \quad 2014$

Decompositions of commutative monoid congruences and binomial ideals

ThOMAS KAHLE and EZRA MiLLER

Locally analytic representations and sheaves on the Bruhat-Tits building

DeEPam Patel, Tobias Schmidt and Matthias Strauch

Complétés universels de représentations de $\mathrm{GL}_{2}\left(\mathbb{Q}_{p}\right)$

Pierre Colmez and Gabriel Dospinescu

On moduli spaces for quasitilted algebras

GRZEGORZ BOBIŃSKI 